\title{
Epidemiological Investigation of an Inhalational Anthrax Patient Traveling for Medical Treatment in Beijing Municipality, China, August 2021
}

\author{
Changying Lin'; Xiangfeng Dou'; Daitao Zhang'; Yinqi Sun²; Huiqiang Han ${ }^{3}$; Chunzhi Chen ${ }^{4} ;$ Xiaojun Zhang'; \\ Shuang $\mathrm{Li}^{1}$; Yanwei Chen ${ }^{1}$; Hongbin Zhang ${ }^{2}$; Bin Wang'; Zhichao Liang ${ }^{1}$; Xin Zhang ${ }^{1}$; Feng Wei ${ }^{3}$; \\ Jiangli Wang'; Feng Liu ${ }^{4}$; Quanyi Wang'; Jian Shi" ${ }^{4, *}$; Peng Yang ${ }^{1, *}$
}

\section{Summary \\ What is already known about this topic? Inhalational anthrax, also known as pulmonary anthrax, is an infectious disease caused by Bacillus anthracis. The patients are usually infected by inhaling aerosolized B. anthracis spores from dead animals or animal products. Compared to cutaneous anthrax, inhalational anthrax is rare and deadly and few cases in China were reported. \\ What is added by this report? \\ This report covers all information of clinical features, laboratory testing, and epidemiological characteristics as well as exposure history of a recent primary inhalational anthrax patient who was seeking medical treatment in Beijing Municipality in August 2021. New laboratory techniques, including second- generation sequencing, polymerase chain reaction, and rapid test for serum antibody, played an important role in the process. \\ What are the implications for public health practice? \\ The information provided in this report, including the correct sample type, epidemiological investigation details, and application of the new diagnostic criteria of anthrax, could assist public health professionals in dealing with anthrax epidemics.}

At $16: 18$ on August 8, 2021, a hospital in Beijing Municipality reported a suspected case of inhalational anthrax. Six hours later, Beijing CDC reported that pleural effusion sample of the case tested positive for nucleic acids of Bacillus anthracis using fluorescent realtime polymerase chain reaction (PCR). On August 13, 1 strain of $B$. anthracis was isolated from dead cattle in the patient's village. On August 19 and 20, 2 samples of pleural effusion collected from the patient with 24hour interval were negative for $B$. anthracis both by real-time PCR and bacterial culture. This met the requirements for discharging anthrax cases from hospital isolation. A total of 127 close contacts of the case in Beijing were quarantined at designated sites or at home for 12 days from the last contact with the patient.

\section{INVESTIGATION AND RESULTS}

The patient was a 46-year-old female from Weichang Manchu and Mongolian Autonomous County, Chengde City, Hebei Province. The patient had sudden chest pain on July 30, 2021 at home with continuous tingling in the right chest, radiating to the right shoulder. The pain worsened when respiring deeply, accompanied by chest tightness, shortness of breath, and asthenia. However, there was no fever, cough, sputum, or hemoptysis. She went to the local county-level and then prefecture-level hospitals on July 31 and was suspected of cardiovascular diseases, such as myocardial infarction and aortic dissection. Fever appeared at $14: 30$ on August 1. After screening negative for severe acute respiratory syndrome coronavirus 2 (SARS-CoV-2) in the fever clinic, the computed tomography (CT) examination at 21:00 showed lesions in the right pulmonary hilar and mediastinum with suspected space-occupying lesions, bilateral pleural effusion, atelectasis in both lungs, and pericardial effusion. The results of two CT examinations by the hospitals in Beijing were similar.

The patient came to Hospital A in Beijing by an ambulance at $02: 00$ on August 3, accompanied by her families. After screening negative for the SARS-CoV-2 in the fever clinic, the patient was given symptomatic anti-inflammatory, analgesic, and antipyretic treatments in the emergency department of Hospital A. Symptoms of pain were relieved. The results of the CT examination showed bilateral pleural effusion, 
atelectasis in both lungs, and space-occupying lesions in the mediastinum.

At 14:00 on August 4, the patient was transferred to Hospital B by a Beijing Emergency Medical Center ambulance. The medical record for admission of the patient showed that she had no erythema, papules, or vesicles on the skin of unknown cause and no fever, nausea, or vomiting. Moist rale was found by lung auscultation and the CT showed lesions in the right pulmonary hilar and mediastinum, with suspected space-occupying lesions, bilateral pleural effusion, atelectasis in both lungs, and pericardial effusion. The CT results were similar to previously reported cases (1-2). Other clinical findings were liver injury and hypoalbuminemia. A total of $200 \mathrm{~mL}$ of hemorrhagic pleural fluid was collected via the closed thoracic drainage method. The patient was treated with imipenem-cilastatinsodium injection and moxifloxacin hydrochloride sodium chloride injection. The seventh group of lymph nodes was positioned by endobronchial ultrasound (EBUS) and punctured, and some purulent secretions were observed in the puncture fluid. Metagenomic sequencing detected sequences of $B$. anthracis in the puncture fluid.

Various samples of the patient were collected on August 8, 2021. The sample of pleural effusion was positive for nucleic acid of $B$. anthracis by fluorescent real-time PCR. Serum antibody against $B$. anthracis was positive by colloidal gold test (Table 1). As a confirmed case, the patient was transferred to the designated infectious disease hospital for quarantine and treatment on the same day. On August 19 and 20, two samples of pleural effusion within a $24 \mathrm{~h}$-interval collected from the case were negative for $B$. anthracis by real-time PCR and bacterial culture.

The patient's home is in the Bashang Grassland, which was a semi-pastoral area, and the patient often had close contact with cattle and sheep. However, the course of the anthrax often progresses rapidly for cattle and sheep and no chronic or carrier states exist. The probability of infection through touching asymptomatic cattle or sheep was estimated to be very low. The patient was also engaged in restaurant operations, so she often visited the meat wholesale market to purchase beef and mutton every two or three days before the disease onset. However, contact with meat or blood of dead animals is more likely to cause cutaneous anthrax instead of inhalational anthrax. The patient had no sign of cutaneous anthrax, no erythema, papule, or verruca, as shown in the medical records of Hospital B, so the probability of infection from exposure to meat from the market was also very low.

The two cattle raised by the patient's brother-in-law died of an unknown illness in the morning of July 26 and were slaughtered at once in the backyard of the patient's house. At the same time, the patient's brother-in-law was told by the doctor that he might have cutaneous anthrax; he called from hospital to stop the slaughtering of the two cattle. The meat, furs, and other parts of the two cattle were disposed outside the village. The slaughter site was immediately disinfected with $1,000 \mathrm{~mL}$ of cresol soap solution, the ground was washed with a high-pressure water gun, and the sewage entered the drainage ditch through a drain. In the afternoon, and the next day, the other two cattle died and were buried directly without slaughter. The sporecontaining droplets produced by high-pressure water gun washing could be the possible cause of the infection of the patient because she had to pass the slaughter site to go to the toilet.

The patient's brother-in-law was a 54-year-old male. He felt itchy on the back of his right hand on July 23 and found a rice grain-sized rash that gradually reddened and became swollen with pain. The site of skin turned black after the ulceration without fever. In the morning of July 26, he went to the county hospital. The doctor suspected cutaneous anthrax but results of stained smear examination for the damaged skin was positive for cocci and no bacilli were detected at the time. The doctor still prescribed levofloxacin infusion therapy for him.

The patient's brother-in-law received levofloxacin

TABLE 1. Test results of the patients' samples on August 8, 2021.

\begin{tabular}{|c|c|c|c|c|c|c|}
\hline \multirow{2}{*}{ Sample type } & \multicolumn{2}{|c|}{ Colloidal gold test } & \multicolumn{3}{|c|}{ Real-time PCR target genes } & \multirow{2}{*}{ Bacterial culture } \\
\hline & Antigen & Antibody & pagA & cap & rpoB & \\
\hline Oropharyngeal swab & Neg & Not tested & Neg & Neg & Neg & Neg \\
\hline Nasopharyngeal swab & $\mathrm{Neg}$ & Not tested & Neg & $\mathrm{Neg}$ & Neg & Neg \\
\hline Pleural effusion & Neg & Not tested & Pos & Pos & Pos & Neg \\
\hline Blood & Neg & Pos & Neg & $\mathrm{Neg}$ & $\mathrm{Neg}$ & Neg \\
\hline
\end{tabular}

Abbreviations: $\mathrm{PCR}=$ polymerase chain reaction; Neg=negative; Pos=positive. 
infusion therapy for 10 days. He went again to the hospital in town due to pain in his right hand. The hospital found his skin damage site on the back of his right hand to be black with eschar and reported the case. On August 9, the Chengde CDC reported realtime PCR negative results for nucleic acid of $B$. anthracis of his skin smear sample, but positive for serum antibody against $B$. anthracis by colloidal gold test. The possible source of infection for the patient's brother-in-law could be the $B$. anthracis spores in the soil. The village is located within a historically anthraxendemic region. The heavy rainfall this year may have caused the spores in the soil to be revealed on the surface and contaminated the grass. The time of infection could be the same as the cattle or when the patient disposed of the sick cattle.

On August 9, 3 samples of furs and 3 samples of beef were collected from the buried dead cattle. The beef samples were positive for nucleic acid of $B$. anthracis and one strain of $B$. anthracis was isolated by bacterial culture from one sample of the beef.

Inhalational anthrax is usually caused by inhaled spores of $B$. anthracis from animal fur, wool, textile mill, or by bioterrorist attack (3). The current case was also related to infected animals because the case was preliminarily attributed to being caused by droplets produced by the high-pressure water gun in the process of washing the grounds where the cattle were slaughtered. In addition, the patient was in poor health and vulnerable to infection. She likely passed the site of slaughtering and became infected.

\section{PUBLIC HEALTH RESPONSE}

The current event of inhalational anthrax was reported as a level IV public health emergency. The patient was quarantined and treated in a single room at the designated infectious hospital. For contact tracing, in accordance with the Anthrax Diagnosis, Treatment, and Management Plan (2005), "family members of the patient, caregivers, and medical staffs who had direct contact with the patient, those who had contact with the excrements of the patient, and individuals who stayed in the same room or stayed with the patient for at least 30 minutes within 5 meters of the patient" should be defined as close contacts. There were 9 family members, 45 medical staff members from various hospitals, 5 medical staff members in the ambulance, 37 hospitalized patients, 30 caregivers of patients, and 1 person collecting clinical samples identified as close contacts. Among them, 9 family members not only had close contact with the patient but also had a history of common environmental exposure in the patient's village. All were quarantined for 12 days from the last contact with the patient. They were required to measure body temperature and report health status everyday. No secondary case was found. The possible contaminated areas in Hospital A and $\mathrm{B}$, as well as the ambulance for transferring the patient were disinfected.

\section{DISCUSSION}

There are still misunderstandings about anthrax. There was no report on person-to-person transmission of inhalational anthrax under natural conditions (4). The medical staff members in local hospitals in Hebei Province and Beijing Municipality had primary personal protection, including working clothes and surgical masks. No infection was observed. Person-toperson transmission has only been reported with cutaneous anthrax, where discharges from skin lesions might be infectious (5). In this report, the fluorescent real-time PCR results of the throat swab and nasopharyngeal swab were negative, while the result of pleural effusion was positive. These findings were consistent with the pathological findings that the anthrax lesions existed mainly in the mediastinum rather than the lungs $(2,6)$. However, currently existing regulations still require quarantine of close contacts of inhalational anthrax cases. Therefore, it is necessary to further update the technical guidelines based on integrating evidence from previous cases and documentation.

Under natural conditions, primary inhalational anthrax is rare and fatal ( 7 ). The droplets aroused by this high-pressure water gun should contain a relatively small number of bacteria. Healthy people washing the ground may not be infected but the patient was not in good health and likely vulnerable. However, people with a history of common exposure were at the same risk, so we suggest that these people need to be quarantined.

The patient's first symptoms were atypical, with no fever in the early stage; the chest pain and tightness was suspected at first to be caused by cardiovascular disease. This perhaps was related to being exposed to particles containing lower levels of $B$. anthracis. The patient's medical history in multiple hospitals showed that no epidemiological exposure history was asked by the doctors, which should be one of the reasons for the misdiagnosis. The physicians should be informed to 
pay more attention to epidemiological history.

The doctors in the local hospitals of Hebei Province showed good awareness of cutaneous anthrax. The doctor in the county-level hospital immediately suspected anthrax and prescribed correct antibiotics for the patient's brother-in-law. Inhalational anthrax was hard to distinguish clinically from other illnesses and was once diagnosed with cardiovascular disease. New techniques, like second generation sequencing would effectively assist the diagnosis. Training of medical staff members with knowledge of anthrax should be carried out.

Beijing has high-quality medical resources; patients from other regions tend to come to Beijing to seek medical treatment. Cases of infectious diseases, such as the 2 cases of pneumonic plague in 2019 may be imported to the city (8). This inhalational anthrax patient entered Beijing by ambulance, so there were no other contacts outside of hospitals. This reminds us that for cases that do not rule out infectious diseases, remote transfer should be further standardized, and the transfer and receiving process should be optimized to ensure that the cases can be treated in time, while at the same time conducive to the prevention and control of infectious diseases.

doi: $10.46234 / \mathrm{ccdcw} 2021.275$

\# Corresponding authors: Shi Jian, shijian_163@163.com; Yang Peng, yangpengcdc@163.com.

\footnotetext{
${ }^{1}$ Beijing Center for Disease Control and Prevention, Beijing, China;
}

2 Hebei Provincial Center for Disease Control and Prevention, Shijiazhuang, Hebei, China; ${ }^{3}$ Chengde Center for Disease Control and Prevention, Chengde, Hebei, China; ${ }^{4}$ Haidian District Center of Disease Control and Prevention, Haidian, Beijing, China; ${ }^{5}$ Weichang County Center for Disease Control and Prevention, Chengde, Hebei, China.

Submitted: August 28, 2021; Accepted: November 19, 2021

\section{REFERENCES}

1. Borio L, Frank D, Mani V, Chiriboga C, Pollanen M, Ripple M, et al. Death due to bioterrorism-related inhalational anthrax: report of 2 patients. JAMA 2001;286(20):2554 - 9. http://dx.doi.org/10.1001/jama. 286.20.2554.

2. Frazier AA, Franks TJ, Galvin JR. Inhalational anthrax. J Thoracic Imaging 2006;21(4):252 - 8. http://dx.doi.org/10.1097/01.rti.0000 213570.71161 .84 .

3. Holty JEC, Bravata DM, Liu H, Olshen RA, McDonald KM, Owens DK. Systematic review: a century of inhalational anthrax cases from 1900 to 2005. Ann Intern Med 2006;144(4):270 - 80. http://dx.doi.org/10. 7326/0003-4819-144-4-200602210-00009.

4. PCB Turnbull. Guidelines for the surveillance and control of anthrax in human and animals. 3rd ed. Geneva: World Health Organization. 1998. https://www.who.int/csr/resources/publications/anthrax/whoemczdi 986text.pdf?ua=1.

5. Beijing Center for Disease Prevention and Control. How people get anthrax. https://www.cdc.gov/anthrax/transmission/index.html. [202011-20].

6. Withers M. USAMRIID's medical management of biological casualties handbook. 8th ed. Frederick: U.S. Army Medical Research Institute of Infectious Diseases. 2014; p. 32-41.

7. Chen WJ, Lai SJ, Yang Y, Liu K, Li XL, Yao HW, et al. Mapping the distribution of anthrax in mainland China, 2005-2013. PLoS Negl Trop Dis 2016;10(4):e0004637. http://dx.doi.org/10.1371/journal.pntd. 0004637.

8. Zhou HJ, Guo SB. Two cases of imported pneumonic plague in Beijing, China. Medicine 2020;99(44):e22932. http://dx.doi.org/10.1097/md. 0000000000022932 . 